\title{
Thermal history of the early Universe and primordial gravitational waves from induced scalar perturbations
}

\author{
Fazlollah Hajkarim* and Jürgen Schaffner-Bielich $\oplus^{\dagger}$ \\ Institut für Theoretische Physik, Goethe Universität, \\ Max von Laue Straße 1, D-60438 Frankfurt am Main, Germany
}

(Received 4 November 2019; accepted 29 January 2020; published 14 February 2020)

\begin{abstract}
We study the induced primordial gravitational waves (GWs) coming from the effect of scalar perturbation on the tensor perturbation at the second order of cosmological perturbation theory. We use the evolution of the standard model degrees of freedom with respect to temperature in the early Universe to compute the induced gravitational waves background. Our result shows that the spectrum of the induced GWs is affected differently by the standard model degrees of freedom than the GWs coming from the first-order tensor perturbation. This phenomenon is due to the presence of scalar perturbations as a source for tensor perturbations, and it is effective around the quark gluon deconfinement and electroweak transition. In case of considering a scalar spectral index larger than 1 at small scales or a non-Gaussian curvature power spectrum, this effect can be observed by gravitational wave observatories.
\end{abstract}

DOI: 10.1103/PhysRevD.101.043522

\section{INTRODUCTION}

The first observation of a gravitational wave (GW) event from the merger of binary black holes and binary neutron stars by laser interferometer gravitational-wave observatory (LIGO) opened a new way for us to understand the Universe [1-3]. Before, using electromagnetic waves, we could see the physical phenomena in the Universe; however, now, somehow, we can listen to them in case they produce gravity waves. Beyond the GWs produced by astrophysical events at the recent era of cosmic evolution, it would be interesting to listen to the early moments of the Universe to detect the predicted GW by cosmological and particle physics models $[4,5]$. The inflationary scenario as a theory to explain the moments after the big bang and explain the current cosmological observations can produce that kind of primordial gravitational waves (PGWs) $[6,7]$. In principle, if we have detectors of high sensitivity, one can observe such PGWs. However, their detection depends on the first-order tensor to scalar ratio "r," which appears directly in the predicted observable relic density of tensor perturbation at the first order of cosmological perturbation theory. Since the cosmic microwave background (CMB) experiments like Planck have only put an upper bound on the tensor-to-scalar ratio and

\footnotetext{
"hajkarim@th.physik.uni-frankfurt.de

†schaffner@astro.uni-frankfurt.de
}

Published by the American Physical Society under the terms of the Creative Commons Attribution 4.0 International license. Further distribution of this work must maintain attribution to the author(s) and the published article's title, journal citation, and DOI. Funded by SCOAP ${ }^{3}$. some theoretical predictions assume very tiny values close to zero for this quantity, it motivates us to consider the secondorder terms in the cosmological perturbation theory induced by scalar perturbations. This has been done in the literature [8-11] and gained much interest recently [12-27] due to the first direct discovery of GW signals from binary mergers.

The predicted shape for the induced spectrum using the standard model (SM) equation of state at different scales or frequency and the evolution of the induced tensor power spectrum should be roughly like the first-order one. However, this has not been checked numerically, and the goal of this paper is to do exactly this. Studying induced PGW is also interesting when there is non-Gaussianity on scales smaller than the CMB $[20,28]$. This can boost the spectrum of induced PGW to large values of the relic density which might be accessible by near future experiments [18-20] especially by pulsar timing arrays. Also, it would be one of the real chances to test particle physics models and the early Universe cosmology based on our current data from CMB and the standard model of particle physics and $\Lambda \mathrm{CDM}$ cosmological constant and cold dark matter in the case in which tensor-to-scalar perturbation ratio is much smaller than the current bound from Planck [29] or from future CMB experiments like CMBS4 [30].

Currently, GW experiments can mostly detect the GW spectrum from astrophysical events with a strong enough amplitude. However, future detectors will be able to probe much smaller signatures from possible phase transitions and the PGW in the early Universe cosmology. Some of these planned GW missions are the Laser Interferometer Space Antenna (LISA) [31], the Einstein Telescope [32], the Deci-Hertz Interferometer Gravitational Wave 
Observatory (DECIGO and B-DECIGO) [33,34], the Big Bang Observatory [35], the Square Kilometer Array (SKA) telescope [36], the North American Nanohertz Observatory for Gravitational Waves [37], and the European Pulsar Timing Array [38]. Moreover, LIGO can be sensitive to the predicted GWs for the early Universe depending on the model $[39,40]$.

For a pre-big-bang-nucleosynthesis $(\mathrm{BBN})$ cosmology which is dominated by some matter with an equation of state different from radiation, the spectrum of the first order and the induced PGW will be different as studied in Refs. [9,12,13,15,16,41-45]. Such models will not be the focus of the current paper.

The value of secondary produced PGW depends on the amplitude of scalar perturbations which is observed at the CMB scale [29]. Since the scalar spectral index at large scales is highly constrained by CMB and is close to 1 , it is not expected to deviate significantly from a flat scalar power spectrum for the standard case. However, it might be different at smaller scales, e.g., in the pre-BBN era. This makes the induced PGW different from the first-order one, which has different free parameters, i.e., the scalar-totensor ratio and the tensor spectral index $[41,46,47]$.

In the next section (Sec. II), we investigate the thermal history of the early Universe including SM particles. In Sec. III, the set of equations related to first-order and secondorder perturbations and the induced PGW will be discussed. We compute the relic density of the induced PGW for the scale-invariant and the scale-dependent scalar perturbations in Sec. IV. Finally, we summarize our results in Sec. V.

\section{THERMAL HISTORY OF THE EARLY UNIVERSE AND STANDARD MODEL PARTICLES}

The effect of standard model degrees of freedom (d.o.f.) on the (first-order) PGW especially around the quark gluon deconfinement and electroweak transition are studied in the literature [41,46-49]. The evolution of d.o.f. influences the relation between the scale factor and the temperature in the early Universe cosmology $[49,50]$. However, this can be interpreted as an evolution of the equation-of-state parameter. The equation-of-state parameter and the speed of sound can be driven by other thermodynamics variables as

$$
\omega=\frac{p_{\mathrm{tot}}}{\rho_{\mathrm{tot}}}, \quad c_{s}^{2}=\frac{\partial p_{\mathrm{tot}}}{\partial \rho_{\mathrm{tot}}}, \quad p_{\mathrm{tot}}=T s_{\mathrm{tot}}-\rho_{\mathrm{tot}},
$$

where the energy density and the entropy density are defined as

$$
\rho_{\mathrm{tot}}=\frac{\pi^{2}}{30} g_{\mathrm{eff}}(T) T^{4}, \quad s_{\mathrm{tot}}=\frac{2 \pi^{2}}{45} h_{\mathrm{eff}}(T) T^{3}
$$

and the effective relativistic d.o.f. for the energy and the entropy density are denoted by $g_{\text {eff }}$ and $h_{\text {eff }}$. The contribution of every relativistic fermion (boson) to the effective DoF is

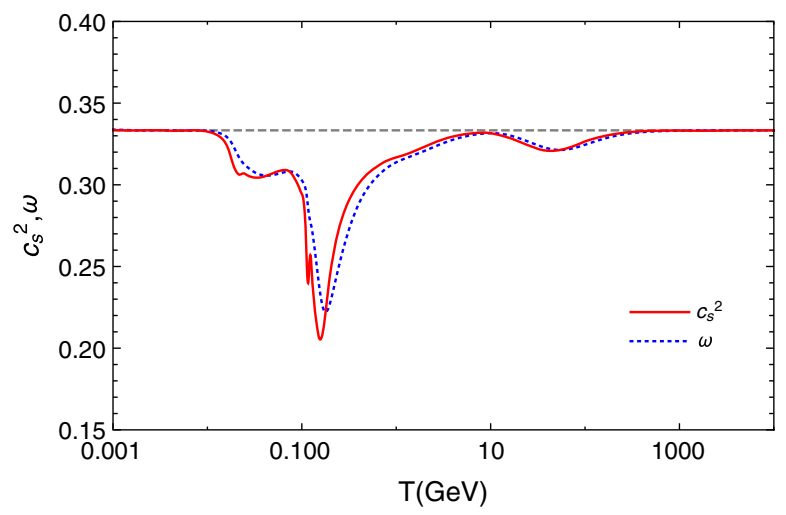

FIG. 1. The speed of sound $c_{s}^{2}$ (solid red curve) and the equation-of-state parameter $\omega$ (blue dotted curve) with respect to temperature $T$ including all the SM d.o.f. at relevant temperatures using the result of Ref. [50]. The dashed line shows the ideal gas case $c_{s}^{2}=\omega=1 / 3$.

$7 / 8$ (1). For radiation or any ideal relativistic fluid, $c_{s}^{2}=\omega=1 / 3$. We use the result of Ref. [50] to compute the equation-of-state parameter and speed of sound for SM d.o.f. as plotted in Fig. 1. This figure shows that around temperatures of $150 \mathrm{MeV}$ and $100 \mathrm{GeV}$, due to QCD and electroweak crossover transitions, $\omega$ and $c_{s}^{2}$ will be smaller than $1 / 3$. This happens since the interaction between particles in the thermal bath of the early Universe deviates from an ideal relativistic fluid. Every extra d.o.f., e.g., due to dark matter or any beyond the SM physics in the early Universe can also lead to small effects on these quantities. Especially, considering a highly populated sector like supersymmetry can modify the result of Fig. 1 and add a new valley at temperatures around $1 \mathrm{TeV}$ or higher depending on the scale of supersymmetry (see Refs. $[47,51,52]$ for supersymmetric DoF and PGW).

\section{TENSOR AND SCALAR PERTURBATION EQUATIONS}

At second order of the cosmic perturbation theory, one can consider the metric $[8,10,12]$

$$
\begin{aligned}
\mathrm{d} s^{2}= & g_{\mu \nu} \mathrm{d} x^{\mu} \mathrm{d} x^{\nu}=-a^{2}(1+2 \Phi) \mathrm{d} \eta^{2} \\
& +a^{2}\left((1-2 \Psi) \delta_{i j}+\frac{1}{2} h_{i j}\right) \mathrm{d} x^{i} \mathrm{~d} x^{j},
\end{aligned}
$$

where $a$ is the scale factor and $\eta$ is the conformal time. Assuming the metric does not contain anisotropic stress $(\Phi=\Psi)$, the scalar perturbation and tensor perturbation are denoted by $\Phi$ and $h$, respectively. The metric in Eq. (3) is considered in Newtonian gauge. Here, we mainly follow Ref. [12] for the evolution equations of induced scalar and tensor perturbations. The evolution of the tensor power spectrum sourced from scalar perturbation can be obtained by solving the equation in Fourier space $[8-10,12]$ 


$$
h_{\vec{k}}^{\prime \prime}(\eta)+2 \mathcal{H} h_{\vec{k}}^{\prime}(\eta)+k^{2} h_{\vec{k}}(\eta)=4 S_{\vec{k}}(\eta),
$$

where the conformal Hubble parameter is denoted by $\mathcal{H}=a H$ and the derivative with respect to the conformal time is denoted by ${ }^{\prime}=d / d \eta$. The Hubble parameter can be evaluated from the Friedmann equation $H^{2}=\left(8 \pi / 3 M_{\mathrm{Pl}}^{2}\right) \rho_{\mathrm{tot}}$, with $M_{\mathrm{Pl}}$ being the Planck mass. Also, the entropy conservation in the early Universe is considered. ${ }^{1}$ To consider the whole evolution of DoF for finding $a(\eta)$, one should solve $a^{\prime}=a^{2} H$ numerically assuming $a_{0}=a\left(\eta_{0}\right)=1$ at today.

The source term on the right-hand side of Eq. (4) assuming $\Phi=\Psi$ can be evaluated as $[8-10,12]$

$$
\begin{aligned}
S_{\vec{k}}= & \int \frac{\mathrm{d}^{3} l}{(2 \pi)^{3 / 2}} \epsilon_{i j}(\vec{k}) l_{i} l_{j}\left(2 \Phi_{\vec{l}} \Phi_{\vec{k}-\vec{l}}\right. \\
& \left.+\frac{4}{3(1+\omega)}\left(\mathcal{H}^{-1} \Phi_{\vec{l}}^{\prime}+\Phi_{\vec{l}}\right)\left(\mathcal{H}^{-1} \Phi_{\vec{k}-\vec{l}}^{\prime}+\Phi_{\vec{k}-\vec{l}}\right)\right),
\end{aligned}
$$

where the polarization tensor for GW is shown by $\epsilon_{i j}(\vec{k})$. In Eq. (5), the terms depending on $c_{s}^{2}$ cancel out so that the expression depends only on $\omega$ explicitly. We use the Green's function method to solve the differential equation for tensor perturbations. Then, the solution in the comoving frame will be [12]

$$
a(\eta) h_{\vec{k}}(\eta)=4 \int_{\eta_{\mathrm{i}}}^{\eta} \mathrm{d} \tilde{\eta} \mathcal{G}_{\vec{k}}(\eta, \tilde{\eta}) a(\tilde{\eta}) S_{\vec{k}}(\tilde{\eta})
$$

where we make use of the Green's function as the solution of the following equation $[8,12]$ :

$$
\mathcal{G}_{\vec{k}}^{\prime \prime}(\eta, \tilde{\eta})+\left(k^{2}-\frac{a^{\prime \prime}(\eta)}{a(\eta)}\right) \mathcal{G}_{\vec{k}}(\eta, \tilde{\eta})=\delta(\eta-\tilde{\eta}) .
$$

For a fixed $\omega$, the scale factor evolves as $a \propto \eta^{\frac{2}{3 \omega+1}}$. Then, Eq. (7) will be given by

$$
\begin{aligned}
& \mathcal{G}_{\vec{k}}^{\prime \prime}(\eta, \tilde{\eta})+\left(k^{2}-\left[\frac{2}{3 \omega+1}\right]\left[\frac{2}{3 \omega+1}-1\right] \frac{1}{\eta^{2}}\right) \mathcal{G}_{\vec{k}}(\eta, \tilde{\eta}) \\
& \quad=\delta(\eta-\tilde{\eta}) .
\end{aligned}
$$

To compute the Green's function, we use the method of Ref. [8] in both cases of constant and evolving $\omega$. If the functions $\mathcal{G}_{1}$ and $\mathcal{G}_{2}$ are two homogeneous solutions of Eq. (7) for a mode $k$, then the Green's function will be defined as

\footnotetext{
${ }^{1}$ To find the evolution of temperature with respect to scale factor in the standard cosmology after reheating, the entropy conservation should be assumed. This can be taken into account by the relation $\frac{d T}{d a}=-\frac{\frac{T}{a}}{1+\frac{T}{3 h_{\text {eff }}(T)} d h_{\text {eff }}(T)}$.
}

$$
\mathcal{G}(\eta, \tilde{\eta})=\frac{\mathcal{G}_{1}(\eta) \mathcal{G}_{2}(\tilde{\eta})-\mathcal{G}_{1}(\tilde{\eta}) \mathcal{G}_{2}(\eta)}{\mathcal{G}_{1}^{\prime}(\tilde{\eta}) \mathcal{G}_{2}(\tilde{\eta})-\mathcal{G}_{1}(\tilde{\eta}) \mathcal{G}_{2}^{\prime}(\tilde{\eta})}
$$

where in practice we compute the numerical form of this function [assuming $\mathcal{G}_{1}(\eta)=0, \mathcal{G}_{1}^{\prime}(\eta)=1 ; \mathcal{G}_{2}(\eta)=1$, $\mathcal{G}_{2}^{\prime}(\eta)=0$ for the initial conditions at superhorizon scales] for each mode $k$ or GW frequency $f=2 \pi / k$. We find two different numerical solutions (using the mentioned initial conditions) of the second-order differential equation in (7) or (8). Then, we use Eq. (9) to define the numerical (interpolated) Green's functions, which we will use in the calculation of the induced tensor power spectrum.

The evolution of scalar perturbations in cosmology can be described with the differential equation $[12,53]$

$$
\begin{aligned}
\Phi_{\vec{k}}^{\prime \prime} & +3 \mathcal{H}\left(1+c_{\mathrm{s}}^{2}\right) \Phi_{\vec{k}}^{\prime}+\left(2 \mathcal{H}^{\prime}+\left(1+3 c_{\mathrm{s}}^{2}\right) \mathcal{H}^{2}+c_{\mathrm{s}}^{2} k^{2}\right) \Phi_{\vec{k}} \\
& =\frac{a^{2}}{2} \tau \delta \mathcal{S},
\end{aligned}
$$

where $\delta p=c_{s}^{2} \delta \rho+\tau \delta \mathcal{S}$. By assuming $c_{s}^{2}=\omega=$ const. and vanishing entropy perturbation in cosmology $\delta \mathcal{S}=0$, one gets [12]

$$
\Phi_{\vec{k}}^{\prime \prime}(\eta)+\frac{6(1+\omega)}{(1+3 \omega) \eta} \Phi_{\vec{k}}^{\prime}(\eta)+\omega k^{2} \Phi_{\vec{k}}(\eta)=0 .
$$

In the limit $c_{s}^{2} \rightarrow \omega$, our numerical results match with the analytical solution of Eq. (11) as given in Refs. [8,12]. However, for a precise numerical calculation, one should distinguish between the speed of sound $c_{s}^{2}$ and the equation-of-state parameter $\omega$ as shown in Fig. 4 and consider them separately in the computation. The correlation function for curvature perturbations is derived as shown in Ref. [12] and gives

$$
\left\langle\phi_{\vec{k}} \phi_{\vec{k}}\right\rangle=\delta(\vec{k}+\overrightarrow{\bar{k}}) \frac{2 \pi^{2}}{k^{3}}\left(\frac{3[1+\omega]}{5+3 \omega}\right)^{2} \mathcal{P}_{\mathcal{R}}(k),
$$

where the superhorizon value of $\phi_{\vec{k}}$ is evaluated from the relation $\Phi_{\vec{k}}=\Phi(k \eta) \phi_{\vec{k}}$. The transfer function $\Phi(k \eta)$ reaches 1 before the moment of horizon crossing $\left(k=a_{h c} H_{h c}\right)$. We will consider the evolution of the d.o.f. of SM in the result of Eq. (10) and other relevant equations in the following.

The induced tensor power spectrum from scalar perturbations is determined via $[8,10,12]$

$$
\begin{aligned}
\mathcal{P}_{T}(\eta, k)= & 4 \int_{0}^{\infty} \int_{|1-v|}^{1+v} \mathrm{~d} v \mathrm{~d} u\left[\frac{4 v^{2}-\left(1+v^{2}-u^{2}\right)^{2}}{4 v u}\right]^{2} \\
& \times \mathcal{I}^{2}(v, u, x) \mathcal{P}_{\mathcal{R}}(k v) \mathcal{P}_{\mathcal{R}}(k u),
\end{aligned}
$$

where $v=|\vec{l}| /|\vec{k}|$ and $u=|\vec{k}-\vec{l}| /|\vec{k}|$. In Eq. (13), the definition of the function $\mathcal{I}$ reads $[10,12]$ 


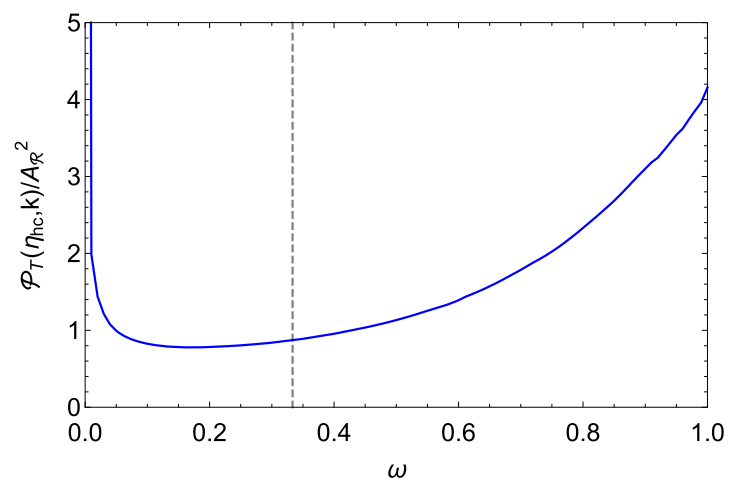

FIG. 2. Evolution of the scaled tensor perturbation at horizon crossing with respect to a constant equation-of-state parameter $\omega$ of the background fluid (blue solid curve). The vertical gray dashed line shows the ideal gas case $\omega=1 / 3$.

$$
\mathcal{I}(v, u, x)=\int_{0}^{x} \mathrm{~d} \tilde{x} \frac{a(\tilde{\eta})}{a(\eta)} k \mathcal{G}_{k}(\eta, \tilde{\eta}) f(v, u, \tilde{x})
$$

where the source function $f$ based on the equation-of-state parameter $\omega$ and transfer functions $\Phi$ is obtained from $[10,12]$

$$
\begin{aligned}
f(v, u, \tilde{x})= & \frac{6(\omega+1)}{3 \omega+5} \Phi(v \tilde{x}) \Phi(u \tilde{x}) \\
& +\frac{12(\omega+1)}{(3 \omega+5)^{2} \mathcal{H}}\left[\partial_{\tilde{\eta}} \Phi(v \tilde{x}) \Phi(u \tilde{x})+\partial_{\tilde{\eta}} \Phi(u \tilde{x}) \Phi(v \tilde{x})\right] \\
& +\frac{12(1+\omega)}{(3 \omega+5)^{2} \mathcal{H}^{2}} \partial_{\tilde{\eta}} \Phi(v \tilde{x}) \partial_{\tilde{\eta}} \Phi(u \tilde{x}) .
\end{aligned}
$$

The terms depending on $c_{s}^{2}$ for the case $\Phi=\Psi$ in the original equation of Refs. $[8,10]$ cancel out. Then, only the terms on the right-hand side of Eq. (15) are left. By changing the variables $u$ and $v$ to $u=(p+q+1) / 2$ and $v=(p-q+1) / 2$ [12], the numerical solution of the tensor power spectrum in Eq. (13) turns out to be computationally faster.

For different values of $\omega$ shown in Fig. 3, we derived the Green's function and $\Phi$ from Eqs. (9) and (11) and computed then the function $f$ from Eq. (15). Then, the function $\mathcal{I}$ was computed analytically from Eq. (14) and used in Eq. (13) for numerical integration. We also checked them with the complete numerical solution, and both of the solutions match each other. As an example, the Green's function for $\omega=1 / 3$ considering $a \propto \eta$ and $a^{\prime \prime}=0$ from Eq. (8) can be evaluated as $\mathcal{G}_{k}(\eta, \tilde{\eta})=\sin (\eta-\tilde{\eta}) / k$ [12]. Also, the scalar transfer function for a radiationlike fluid by solving Eq. (11) and assuming $(\Phi(k \eta \rightarrow 0) \rightarrow 1)$ can be shown to be $[12,54]$

$$
\Phi(k \eta)=\frac{9}{(k \eta)^{2}}\left[\frac{\sin (k \eta / \sqrt{3})}{k \eta / \sqrt{3}}-\cos (k \eta / \sqrt{3})\right] .
$$

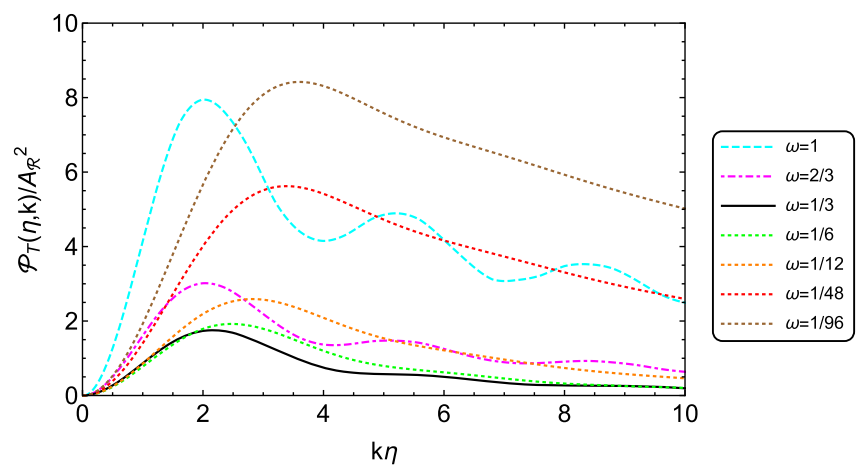

FIG. 3. Evolution of the scaled tensor power spectrum $\mathcal{P}_{T}(\eta, k) / A_{\mathcal{R}}^{2}$ with respect to $k \eta$ for different values of $\omega$ is shown. As $\omega \rightarrow 0$, the tensor power spectrum diverges as the upper limit of first integral in Eq. (13) becomes infinite [12].

The explicit form of the function $\mathcal{I}$ is complicated and long. It will not be presented here (see Refs. [10,12]). In Ref. [12], the limits of the function $\mathcal{I}$ in a radiationdominated Universe are found to be $\mathcal{I}(u, v, x \rightarrow 0) \approx x^{2} / 2$ and $\mathcal{I}(u, v, x \rightarrow \infty) \approx \mathcal{I}(u, v) / x^{2}$. Using the latter limit, the evolution of the tensor power spectrum is estimated to be $\mathcal{P}_{T}(\eta, k) / A_{\mathcal{R}}^{2} \approx 19.7 / x^{2}$ [12]. This expression is vastly used afterward to estimate the value of the GW relic density of today. However, the value 19.7 does not match the results for $\omega=1 / 3$ shown by the solid black line in Fig. 3 based on a complete numerical calculation. This will be explained in more detail in the next section.

\section{PRIMORDIAL GRAVITATIONAL WAVES AND EXPERIMENTAL CONSTRAINTS}

The scale-invariant scalar power spectrum defined in Eq. (12) can be written as [55]

$$
\mathcal{P}_{\mathcal{R}}(k)=A_{\mathcal{R}}
$$

Moreover, the power law scalar power spectrum can be defined as [55]

$$
\mathcal{P}_{\mathcal{R}}(k)=A_{\mathcal{R}}\left(\frac{k}{\tilde{k}}\right)^{n_{s}-1}
$$

with the pivot scale $\tilde{k}=0.05 \mathrm{Mpc}^{-1}$ and the scalar spectral index $n_{s}$.

From Planck satellite observations, the value of the scalar spectral index has been determined to be $n_{s}=0.965 \pm$ 0.004 [29]. Also, the value of the curvature perturbation amplitude at the CMB scale is $A_{\mathcal{R}} \simeq 2.1 \times 10^{-9}$ [29]. In addition, the scale-invariant tensor power spectrum at first order is defined as [55]

$$
\mathcal{P}_{T}^{(1)}=A_{T}
$$


Then, the tensor-to-scalar ratio at first order is [55]

$$
r=\frac{A_{T}}{A_{\mathcal{R}}} .
$$

The current limit from the Planck Collaboration is $r \lesssim$ 0.07 [29].

In Fig. 2, we assume that the parameter $\omega$ is fixed and constant in the calculation of Eqs. (7), (11), and (13). The scaled tensor power spectrum [scaled by the square of the scale-invariant curvature power spectrum: $\left.\mathcal{P}_{T}\left(\eta_{h c}, k\right) / A_{\mathcal{R}}^{2}\right]$ at horizon crossing is computed for the range of $0<\omega<1$, where the minimum of the tensor power spectrum appears at $\omega_{\min }=0.175$. As Fig. 2 shows and as it is analytically calculated in Refs. $[9,12,45]$, if $\omega \rightarrow 0$, the tensor power spectrum diverges, $\mathcal{P}_{T}\left(\eta \geq \eta_{h c}, k\right) \rightarrow \infty$. The reason is that in a matter-dominated era the scalar perturbation becomes constant and the function $\mathcal{I}^{2}$ at late times will have a constant value. Since the other terms in the integrand of Eq. (13) are nonlinear and the integration is effectively done over the modes from very small to very large modes, the result diverges. This happens when one assumes the matterdominated era might last forever; however, this might not be the realistic case in standard and nonstandard cosmologies. The period of matter domination can be limited; then, it should start and end at some specific modes [changing the limits of integration in Eq. (13)]. Then, the second-order tensor power spectrum for the matter-dominated epoch becomes larger than other cases and not divergent. The final result for $\mathcal{P}_{T}\left(\eta_{h c}, k\right) / A_{\mathcal{R}}^{2}$ in the range $0 \leq k \eta \leq 10$ and different $\omega$ 's is shown in Fig. 3. The function $\mathcal{I}$ inside the integral of Eq. (13) is highly oscillatory. It gets its maximum value slightly after horizon crossing $(2 \lesssim k \eta \lesssim 4$ depending on the value of $\omega$ ), then decays proportional to $1 /(k \eta)^{2}$ during radiation domination [12].

For the induced tensor perturbation in the computation of the tensor power spectrum after horizon crossing until today, the WKB method (which considers the numerical value of the transfer function and its first derivative to match the approximate analytical solution of the transfer function) cannot be used to match the result at some point and then extend it until today, since (to the best of our knowledge) there is not any well-defined shape of the transfer function during radiation domination for the induced PGW like $A \sin (\delta+k \eta) / k \eta$ as assumed in Refs. [41,46,47] for the first-order PGW.

The power spectrum, shown in Fig. 3, increases after horizon crossing to a maximum value, then dilutes with the expansion of the Universe. The numerical calculation of such a power spectrum is difficult and time consuming since it includes various numerical integrations over oscillatory functions. In practice, it is very difficult to solve the integral of Eq. (13) for a large range of $k \eta$ from zero to today $k \eta_{0}$. So, one should calculate until horizon crossing or until the peak for $\omega=1 / 3$ in Fig. 3, then use $1 /(k \eta)^{2}$ as the dilution factor due to the expansion of the Universe.

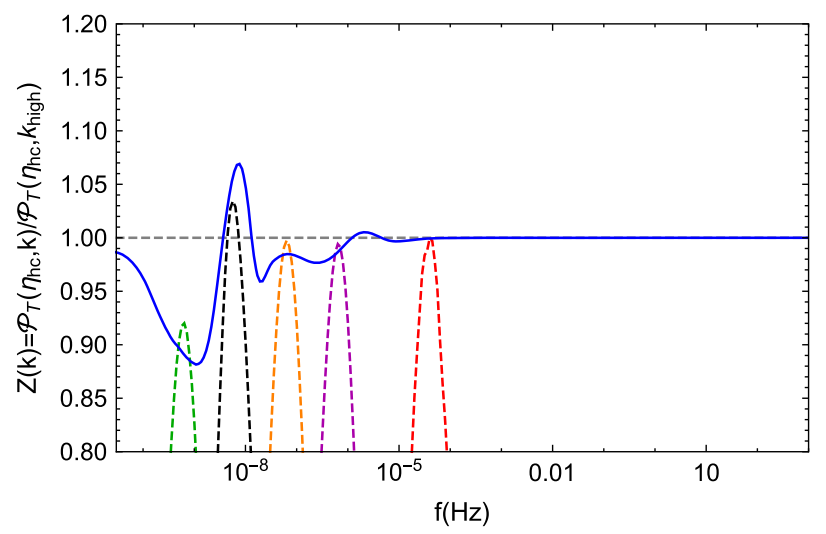

FIG. 4. The correction factor $Z(k)=\mathcal{P}_{T}\left(\eta_{h c}, k\right) / \mathcal{P}_{T}\left(\eta_{h c}, k_{\text {high }}\right)$ on the scalar-induced gravitational waves due to the evolution of standard model d.o.f., which is scaled to the value at high frequencies where $\omega \rightarrow 1 / 3$ well above the electroweak transition. The solid blue line includes the thermal history using all the SM particles. The dashed gray straight line denotes a radiationlike equation of state with $\omega=1 / 3$. The correction factor for nonGaussianity peaks around frequencies $\bar{k} / 2 \pi=\bar{f}=1.6 \times 10^{-9} \mathrm{~Hz}$ (green), $1.6 \times 10^{-8} \mathrm{~Hz}$ (black), $1.6 \times 10^{-7} \mathrm{~Hz}$ (orange), $1.6 \times$ $10^{-6} \mathrm{~Hz}$ (violet), and $10^{-4} \mathrm{~Hz}$ (red) and is plotted in colored dashed curves for the choice of $\left[\mathcal{F}_{N L}^{2}, A_{G}\right]=\left[10,10^{-2}\right]$.

The tensor power spectrum can also be defined in terms of the transfer function $\mathcal{P}_{T}\left(\eta_{h c}, k\right) \propto \mathcal{T}^{\prime}\left(\eta_{h c}, k\right)^{2}=$ $k^{2} \mathcal{T}\left(\eta_{h c}, k\right)^{2}$. It scales like $1 / a^{2}$ after horizon crossing. For the radiation-domination case (without the change of DoF), the scaled tensor power spectrum in Fig. 3 at horizon crossing and at its first peak $\left(\tilde{x}_{1 \mathrm{st} \text {,peak }} \simeq 2.17\right)$ are $\mathcal{P}_{T}\left(\eta_{h c}, k\right) / A_{\mathcal{R}}^{2} \simeq 0.87$ and $\mathcal{P}_{T}\left(\eta_{1 \text { st,peak }}, k\right) / A_{\mathcal{R}}^{2} \simeq 1.75$, respectively $\left[\mathcal{P}_{T}\left(\eta_{1 \text { st,peak }}, k\right) / \mathcal{P}_{T}\left(\eta_{h c}, k\right) \simeq 2\right]$.

It is expected that the evolution of the DoF in the early Universe plays a role in the computation of Eqs. (7), (10), and (13), so we checked it. We have included all DoF of the SM to find the scaled tensor power spectrum $\mathcal{P}_{T}\left(\eta_{h c}, k\right) / A_{\mathcal{R}}^{2}$. Then, the shape of the spectrum will have a correction factor, shown in Fig. 4, due to the evolution of different functions inside the integral of Eq. (13) by a change of the DoF over time. This fact originates from the combination of all functions in the integral $a(\eta) / a(\tilde{\eta})$, $f(u, v, k \tilde{\eta})$, and $\mathcal{G}(\eta, \tilde{\eta})$ due to carrying the retarded effects from previous times. In practice, an analytical formula to show the explicit form of Fig. 4 has not been found. However, it roughly behaves like the evolution of $d \omega / d T$ including the retardation effects due to the Green's function. The inflection points in the correction factor of the secondary PGW spectrum roughly show the points of the crossover transition having the smallest values of $c_{s}^{2}$ and $\omega$ at the relevant frequencies $\left(3 \times 10^{-9}\right.$ and $\left.10^{-6} \mathrm{~Hz}\right)$. In the case in which one adds the supersymmetric DoF or any highly populated sector around the $\mathrm{TeV}$ scale, there will be another inflection point at higher frequencies around $10^{-4} \mathrm{~Hz}$. 

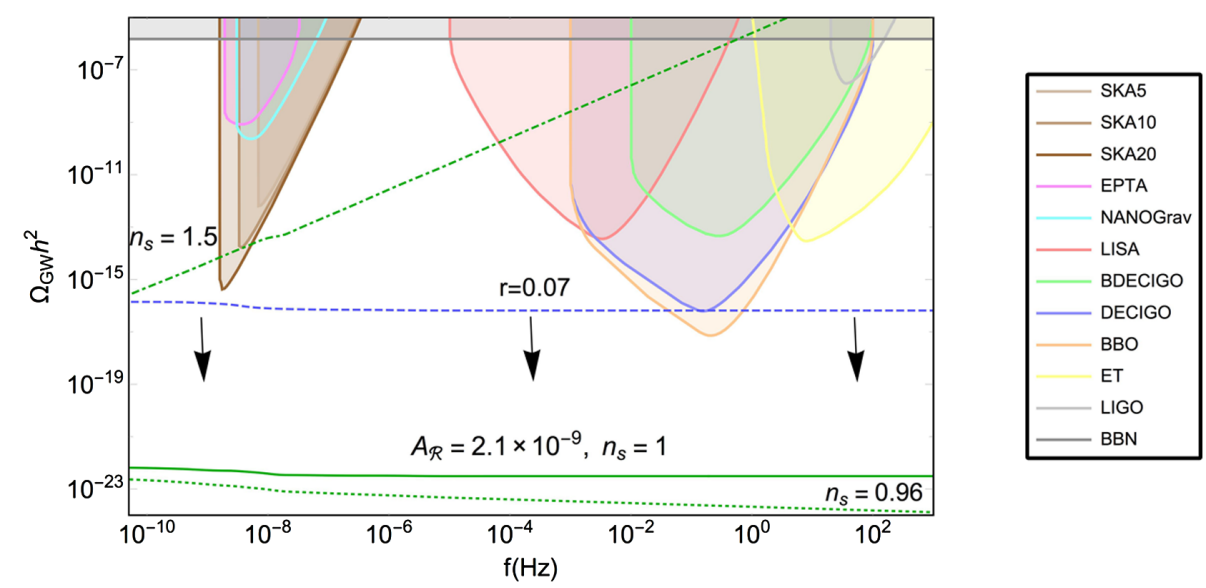

FIG. 5. The spectrum of the PGWs is shown for the scale invariant first-order tensor perturbations (blue dashed line) assuming the maximum value of tensor-to-scalar ratio $r=0.07$ from the upper limit of Planck data [29] and the induced PGWs using the observed value of scalar perturbation amplitude $A_{\mathcal{R}}=2.1 \times 10^{-9}$ assuming the scale invariance (green solid line), a scale dependence with $n_{s}=0.96$ (green dotted line), and $n_{s}=1.5$ (green dot-dashed line). Also, some current and future experimental constraints [31-40] and the BBN bound are shown (see the text for details).

The density of PGW per frequency (wave number) from tensor perturbation at first order in the case of Eq. (4) being source free can be estimated as $[41,46,47]$

$$
\begin{aligned}
\Omega_{G W}^{(1)}\left(\eta_{0}, k\right) h^{2} & =\frac{1}{24}\left(\frac{a_{h c} H_{h c}}{a_{0} H_{0}}\right)^{2}\left\langle\mathcal{P}_{T}^{(1)}\left(\eta_{0}, k\right)\right\rangle_{\mathrm{osc}} \\
& \simeq \frac{1}{24}\left(\frac{h_{\mathrm{eff}}\left(T_{0}\right)}{h_{\mathrm{eff}}\left(T_{h c}\right)}\right)^{\frac{4}{3}}\left(\frac{g_{\mathrm{eff}}\left(T_{h c}\right)}{g_{\mathrm{eff}}\left(T_{0}\right)}\right) \mathcal{P}_{T}^{(1)}(k) \Omega_{\gamma, 0} h^{2},
\end{aligned}
$$

where $\mathcal{P}_{T}^{(1)}(k)=\mathcal{P}_{T}^{(1)}(\eta, k) / \mathcal{T}^{\prime(1)}(\eta, k)^{2}=\mathcal{P}_{T}^{(1)}\left(\eta_{h c}, k\right) /$ $\left(k \mathcal{T}^{(1)}\left(\eta_{h c}, k\right)\right)^{2}$ for the first-order PGW when $\mathcal{T}^{(1)}\left(\eta_{h c}, k\right)^{2}$ is the first-order tensor perturbation transfer function. Here, we focus on frequencies larger than $3 \times 10^{-11} \mathrm{~Hz}$, which are equivalent to temperatures larger than $4 \mathrm{MeV}$. Consequently, we do not consider the effects of neutrinos and photons free streaming, which appear at smaller frequencies [46,56,57] as a new source term on the right-hand side of Eq. (4). The relic PGW today from induced scalar perturbation can be obtained from [54]

$$
\begin{aligned}
\Omega_{\mathrm{GW}}\left(\eta_{0}, k\right) h^{2}= & \frac{1}{24}\left(\frac{a_{h c} H_{h c}}{a_{0} H_{0}}\right)^{2}\left\langle\mathcal{P}_{T}\left(\eta_{0}, k\right)\right\rangle_{\mathrm{osc}} \\
\simeq & \frac{1}{24}\left(\frac{h_{\mathrm{eff}}\left(T_{0}\right)}{h_{\mathrm{eff}}\left(T_{h c}\right)}\right)^{\frac{4}{3}}\left(\frac{g_{\mathrm{eff}}\left(T_{h c}\right)}{g_{\mathrm{eff}}\left(T_{0}\right)}\right) 2 \\
& \times(2.17)^{2} Z(k) \mathcal{P}_{T}\left(\eta_{h c}, k_{\text {high }}\right) \Omega_{\gamma, 0} h^{2},
\end{aligned}
$$

and the correction factor is defined as $Z(k)=\mathcal{P}_{T}\left(\eta_{h c}, k\right) /$ $\mathcal{P}_{T}\left(\eta_{h c}, k_{\text {high }}\right)$, shown in Fig. 4 as blue solid line $\left[\mathcal{P}_{T}\left(\eta_{h c}, k_{\text {high }}\right) / A_{\mathcal{R}}^{2} \simeq 0.87\right]$. The shape of this function completely depends on the details of the functions inside the integral of Eq. (13). The function $Z(k)$ already corrects the spectrum on the order of $10 \%$ especially around the QCD epoch, which is equivalent to a frequency of $3 \times 10^{-9} \mathrm{~Hz}$. As mentioned earlier, the evolution of DoF of SM particles is taken from the results of Ref. [50]. Using the numerical factor $2 \times(2.17)^{2}$ in Eq. (22) from the fact that $\tilde{x}_{1 \text { st,peak }} \simeq 2.17$, the tensor power spectrum from scale-invariant curvature power spectrum during radiation domination evolves as $\left[\mathcal{P}_{T}\left(\eta_{1 \text { st,peak }}, k\right) \tilde{x}_{1 \text { st,peak }}^{2}\right] /$ $\left[\mathcal{P}_{T}\left(\eta_{h c}, k\right) \tilde{x}_{h c}^{2}\right] \times 1 / \tilde{x}^{2} \simeq 2 \times(2.17)^{2} / \tilde{x}^{2}$. The results for the first-order and second-order PGWs are shown in Fig. 5 in addition to the different experimental constraints [31-40] and the BBN bound [12] as outlined in the legend of the plot (the suffix number for the SKA experiment shows the constraints based on the number of years in operation). In case of scale invariance, the relic of the firstorder PGW can have any value from approximately $10^{-16}$ down to the lower limit by the induced PGW of approximately $10^{-23}$ depending on the value of $r$. If $r \lesssim 10^{-9}$, then the scalar-induced contribution on the PGW will be dominating. The scale-dependent induced PGWs for scalar spectral indices of $n_{s}=0.96$ and 1.5 are also plotted in Fig. 5. For the case $n_{s}>1$, the spectrum can be constrained by current and future experiments.

The curvature power spectrum can deviate from a flat shape at scales smaller than the CMB scale, especially if one assumes that there is some Gaussian or non-Gaussian source for it from the curvature bispectrum [18,20,28]. This can enhance the spectrum by some peaks of the relic densities at frequencies accessible by pulsar timing arrays or other experiments. The effect of the evolution of the d.o.f. in the early Universe also appears on the secondary PGW if there is some source of non-Gaussianity [18,20]. To represent this effect, first we assume that the following Gaussian curvature power spectrum (for $k$ in the 

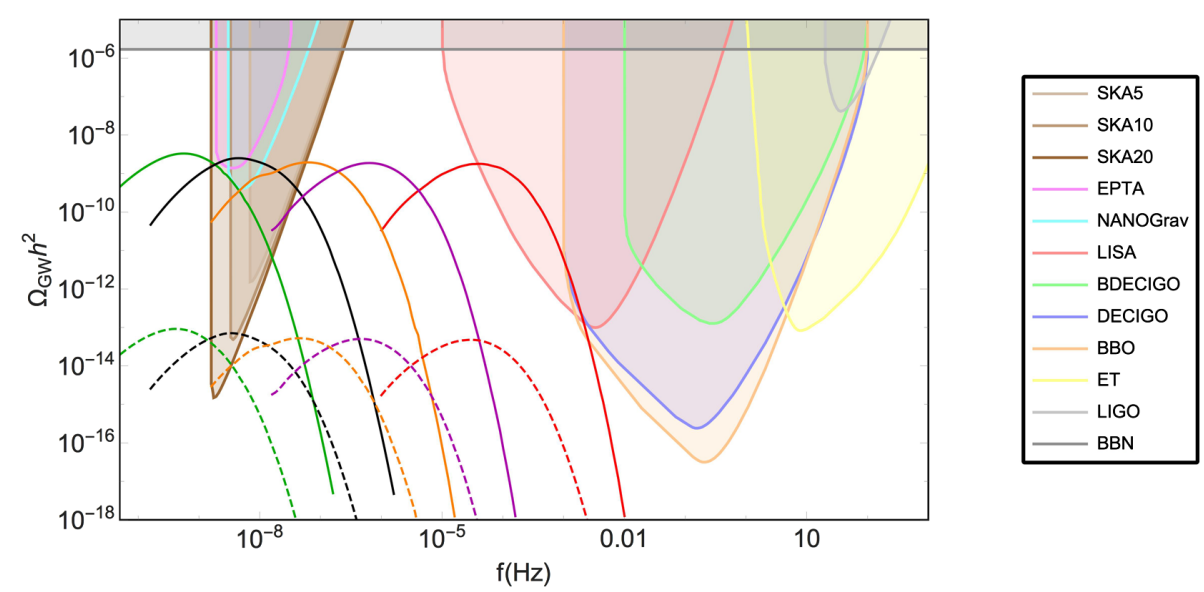

FIG. 6. The induced PGWs from the non-Gaussian scalar power spectrum at small scales is shown for characteristic frequencies $\bar{k} / 2 \pi=\bar{f}=1.6 \times 10^{-9} \mathrm{~Hz}$ (green), $1.6 \times 10^{-8} \mathrm{~Hz}$ (black), $1.6 \times 10^{-7} \mathrm{~Hz}$ (orange), $1.6 \times 10^{-6} \mathrm{~Hz}$ (violet), and $10^{-4} \mathrm{~Hz}$ (red) for two choices of $\left[\mathcal{F}_{N L}^{2}, A_{G}\right]=\left[10,10^{-2}\right]$ (solid curves) and $\left[60,10^{-4}\right]$ (dashed curves).

logarithmic scale) around a specific mode $\bar{k}$ with width $\bar{\sigma}$ [18-20] is parametrized by

$$
\mathcal{P}_{\mathcal{R}, G}=A_{G} \exp \left[-\frac{\ln (k / \bar{k})^{2}}{2 \bar{\sigma}^{2}}\right]
$$

where $A_{G}$ is the Gaussian amplitude that can depend on the characteristic scale $\bar{k}$ and other model-dependent variables. Here, we simply assume it to be constant to test for its observational consequence. By considering the presence of non-Gaussianity in the curvature power spectrum, one gets [18-20]

$$
\begin{aligned}
\mathcal{P}_{\mathcal{R}, N G}(k)= & \mathcal{P}_{\mathcal{R}, G}(k)+\mathcal{F}_{N L}^{2} \\
& \times \int_{0}^{\infty} \int_{|1-v|}^{1+v} \mathrm{~d} v \mathrm{~d} u \frac{\mathcal{P}_{\mathcal{R}, G}(k v) \mathcal{P}_{\mathcal{R}, G}(k u)}{v^{2} u^{2}},
\end{aligned}
$$

where the nonlinear factor for the non-Gaussian curvature perturbation is denoted by $\mathcal{F}_{N L}$. We choose different benchmark points for $\bar{k}$ and $\mathcal{F}_{N L}$ to see how its consequence will be on the PGW. Also, we consider the evolution of DoF to check how it influences the induced PGWs by the non-Gaussian power spectrum. This is also shown in Fig. 4 for the scaled tensor power spectrum assuming $\mathcal{F}_{N L}^{2}=10, A_{G}=10^{-2}$, and $\bar{\sigma}=1$ for different $\bar{k}$ 's. As we expect from previous discussion, the effect of QCD and electroweak transitions is also apparent in this case for different peaks around modes $\bar{k} / 2 \pi=\bar{f}=1.6 \times 10^{-9}$, $1.6 \times 10^{-8}, 1.6 \times 10^{-7}$, and $1.6 \times 10^{-6} \mathrm{~Hz}$. The value of the scaled tensor power spectrum in the mentioned modes is scaled to its value at a high frequency $\bar{f}=10^{-4} \mathrm{~Hz}$. We should emphasize again that this effect is due to the changes of the equation-of-state parameter and DoF during cosmic transitions (see Fig. 1) in the calculation of Eq. (13).
Non-Gaussianity at the CMB scale is constrained by Planck data [58]. However, there are fewer constraints on non-Gaussianity at small scales. To predict a realistic signal for the presence of non-Gaussianity, we assume $A_{G} \mathcal{F}_{N L}^{2} \ll 1[20]$. This causes the second term on the righthand side of Eq. (24) to be smaller than the first term, which means that the non-Gaussian part of the curvature power spectrum is smaller than the Gaussian one. However, the contribution of non-Gaussianity on the second-order PGW peak can be larger than the Gaussian part depending on the values of $\mathcal{F}_{N L}$ and $\bar{\sigma}$ [20]. In Fig. 6, the non Gaussianinduced PGWs for $\left[\mathcal{F}_{N L}^{2}, A_{G}\right]=\left[10,10^{-2}\right]$ and $\left[60,10^{-4}\right]$ for different modes are plotted using Eqs. (22), (23), and (24). For these peaks, pulsar timing array experiments or LISA can be sensitive enough to observe such peaks on the GW background, which can be distinguished from other prediction, e.g., by first-order phase transitions, due to the specific shape of the spectrum. Consequently, the effect of the thermal history of the Universe and the induced tensor spectra from scalar perturbations will be small at the peaks and can provide information about pre-BBN cosmology.

\section{CONCLUSIONS}

The second-order PGWs from scalar perturbations have been studied analytically and by a numerical calculation in the radiation- and early matter-dominated epochs [8-22]. Here, first we focused on studying the effect of the equation-of-state parameter on the induced PGWs during the dominance of radiation or any fluid with $0<\omega<1$ (see Fig. 2). The shape of the tensor spectrum is shown in Fig. 3 for different equation-of-state parameters for $0 \leq k \eta \leq 10$. Moreover, the effect of the d.o.f. of thermal bath particles evolving with temperature on the spectrum of induced PGWs at different frequencies is studied and shown by a correction function $Z(k)\left[Z\left(T_{h c}\right)\right]$ in Eq. (22) and Fig. 4. This function includes the evolution 
of the tensor perturbation and the retarded effects from the scalar Green's function and is particularly important around the QCD and electroweak transitions. It is possible that future experiments do not observe the first-order PGW for a scale-independent tensor spectral index and tiny values of the tensor-to-scalar ratio approximately $10^{-9}$ at first order, based on the current knowledge of $\Lambda \mathrm{CDM}$ cosmology and observations. Then, it is expected that the induced PGWs should be observable when GW experiments can probe the PGW relic down to approximately $5 \times 10^{-23}$ (Fig. 5). Larger values due to non-Gaussianity (Fig. 6) are measurable by the recently planned missions and are distinguishable from astrophysical backgrounds. In a sense, what is calculated here is the combined prediction of general relativity based on just the SM of particle physics using the current CMB observation for the early Universe cosmology.

Principally, one should be able to observe the impact of the thermal history of the early Universe on the induced PGW background from scalar perturbations. Since we have already observed the scalar perturbation at the CMB scale and their effect on structure formation, it would be reasonable to see its indirect impact on the production of induced PGWs at smaller scales. Otherwise, there should be some theoretical model-dependent explanation for it from alternative approaches to the big bang cosmology and inflation or an unknown effect at early epochs that has not been investigated before $[8,10,12,22]$.

Considering the effect of the standard model DoF on the induced PGWs will be influential for the future searches of gravitational waves with cosmological origin, since it helps to improve the theoretical prediction due to different effects for the GW spectra from the early Universe. In addition, studying details of the induced PGWs affects our understanding about the impact of thermal evolution of the SM in the early Universe and pre-BBN cosmology on the GW background. Also, searching for the PGWs can be useful as a cosmic laboratory for the indirect probe of new particles being present in the thermal bath of the early Universe and new physics even without observing any boosted effects on the GW spectrum from the predicted cosmic phase transitions and nonstandard cosmological scenarios.

\section{ACKNOWLEDGMENTS}

F. H. thanks Caner Ünal for useful discussions. Also, he is grateful to the hospitality and support of Galileo Galilei Institute for Theoretical Physics at Florence during the last stages of this work. The authors acknowledge the support by the Deutsche Forschungsgemeinschaft (DFG) through the CRC-TR 211 Project No. 315477589-TRR 211.
[1] B. P. Abbott et al. (LIGO Scientific, Virgo Collaborations), Phys. Rev. Lett. 116, 241103 (2016).

[2] B. P. Abbott et al. (LIGO Scientific, Virgo Collaborations), Phys. Rev. Lett. 116, 061102 (2016).

[3] B. P. Abbott et al. (LIGO Scientific, Virgo Collaborations), Phys. Rev. Lett. 119, 161101 (2017).

[4] C. Caprini and D. G. Figueroa, Classical Quantum Gravity 35, 163001 (2018).

[5] M. Maggiore, Phys. Rep. 331, 283 (2000).

[6] L. P. Grishchuk, Zh. Eksp. Teor. Fiz. 67, 825 (1974) [Sov. Phys. JETP 40, 409 (1975)].

[7] A. A. Starobinsky, JETP Lett. 30, 682 (1979).

[8] D. Baumann, P. J. Steinhardt, K. Takahashi, and K. Ichiki, Phys. Rev. D 76, 084019 (2007).

[9] H. Assadullahi and D. Wands, Phys. Rev. D 79, 083511 (2009).

[10] K. N. Ananda, C. Clarkson, and D. Wands, Phys. Rev. D 75, 123518 (2007).

[11] S. Mollerach, D. Harari, and S. Matarrese, Phys. Rev. D 69, 063002 (2004).

[12] K. Kohri and T. Terada, Phys. Rev. D 97, 123532 (2018).

[13] K. Inomata and T. Nakama, Phys. Rev. D 99, 043511 (2019).

[14] M. Drees and Y. Xu, arXiv:1905.13581.

[15] K. Inomata, K. Kohri, T. Nakama, and T. Terada, J. Cosmol. Astropart. Phys. 10 (2019) 071.
[16] K. Inomata, K. Kohri, T. Nakama, and T. Terada, Phys. Rev. D 100, 043532 (2019).

[17] Y. Lu, Y. Gong, Z. Yi, and F. Zhang, J. Cosmol. Astropart. Phys. 12 (2019) 031.

[18] R.-g. Cai, S. Pi, and M. Sasaki, Phys. Rev. Lett. 122, 201101 (2019).

[19] R.-G. Cai, S. Pi, S.-J. Wang, and X.-Y. Yang, J. Cosmol. Astropart. Phys. 10 (2019) 059.

[20] C. Unal, Phys. Rev. D 99, 041301 (2019).

[21] R.-G. Cai, S. Pi, S.-J. Wang, and X.-Y. Yang, J. Cosmol. Astropart. Phys. 05 (2019) 013.

[22] I. Ben-Dayan, B. Keating, D. Leon, and I. Wolfson, J. Cosmol. Astropart. Phys. 06 (2019) 007.

[23] J.-O. Gong, arXiv:1909.12708.

[24] K. Tomikawa and T. Kobayashi, arXiv:1910.01880.

[25] J. R. Espinosa, D. Racco, and A. Riotto, J. Cosmol. Astropart. Phys. 09 (2018) 012.

[26] C. Fu, P. Wu, and H. Yu, Phys. Rev. D 101, 023529 (2020).

[27] G. Domènech, arXiv:1912.05583.

[28] X. Chen, Adv. Astron. 2010, 1 (2010).

[29] N. Aghanim et al. (Planck Collaboration), arXiv:1807. 06209.

[30] K. Abazajian et al., arXiv:1907.04473.

[31] H. Audley et al. (LISA Collaboration), arXiv:1702.00786.

[32] B. Sathyaprakash et al., Classical Quantum Gravity 29, 124013 (2012); 30, 079501(E) (2013). 
[33] N. Seto, S. Kawamura, and T. Nakamura, Phys. Rev. Lett. 87, 221103 (2001).

[34] S. Sato et al., J. Phys. Conf. Ser. 840, 012010 (2017).

[35] J. Crowder and N. J. Cornish, Phys. Rev. D 72, 083005 (2005).

[36] G. Janssen et al., Proc. Sci., AASKA14 (2015) 037 [arXiv: 1501.00127].

[37] Z. Arzoumanian et al. (NANOGRAV Collaboration), Astrophys. J. 859, 47 (2018).

[38] L. Lentati et al., Mon. Not. R. Astron. Soc. 453, 2577 (2015).

[39] B. P. Abbott et al. (LIGO Scientific, Virgo Collaborations), Phys. Rev. D 100, 061101 (2019).

[40] B. P. Abbott et al. (LIGO Scientific, Virgo Collaborations), Phys. Rev. Lett. 120, 091101 (2018).

[41] N. Bernal and F. Hajkarim, Phys. Rev. D 100, 063502 (2019).

[42] D. G. Figueroa and E. H. Tanin, J. Cosmol. Astropart. Phys. 08 (2019) 011.

[43] T. Opferkuch, P. Schwaller, and B. A. Stefanek, J. Cosmol. Astropart. Phys. 07 (2019) 016.

[44] D. Bettoni, G. Doménech, and J. Rubio, J. Cosmol. Astropart. Phys. 02 (2019) 034.

[45] L. Alabidi, K. Kohri, M. Sasaki, and Y. Sendouda, J. Cosmol. Astropart. Phys. 05 (2013) 033.

[46] K. Saikawa and S. Shirai, J. Cosmol. Astropart. Phys. 05 (2018) 035.
[47] Y. Watanabe and E. Komatsu, Phys. Rev. D 73, 123515 (2006).

[48] S. Schettler, T. Boeckel, and J. Schaffner-Bielich, Phys. Rev. D 83, 064030 (2011).

[49] F. Hajkarim, J. Schaffner-Bielich, S. Wystub, and M. M. Wygas, Phys. Rev. D 99, 103527 (2019).

[50] M. Drees, F. Hajkarim, and E. R. Schmitz, J. Cosmol. Astropart. Phys. 06 (2015) 025.

[51] R. Jinno, T. Moroi, and K. Nakayama, J. Cosmol. Astropart. Phys. 01 (2014) 040.

[52] Y. Cui, M. Lewicki, D. E. Morrissey, and J. D. Wells, J. High Energy Phys. 01 (2019) 081.

[53] V. Mukhanov, Physical Foundations of Cosmology (Cambridge University Press, Oxford, 2005), ISBN 0521563984, http://www-spires.fnal.gov/spires/find/books/www?cl= QB981.M89::2005.

[54] K. Ando, K. Inomata, and M. Kawasaki, Phys. Rev. D 97, 103528 (2018).

[55] D. Baumann, in Physics of the large and the small, TASI 09, proceedings of the Theoretical Advanced Study Institute in Elementary Particle Physics, Boulder, Colorado, USA, 2009 (World Scientific, 2011), pp. 523-686.

[56] S. Weinberg, Phys. Rev. D 69, 023503 (2004).

[57] B. A. Stefanek and W. W. Repko, Phys. Rev. D 88, 083536 (2013).

[58] Y. Akrami et al. (Planck Collaboration), arXiv:1905.05697. 